\title{
Performance Comparison of Different STBC codes in the Presence of IQ Imbalances in Mobile Communications
}

\author{
B. Banitalebi \\ Telecom. Company of Iran \\ Iran - Shahrekoard \\ bbanitalebi@yahoo.com
}

\begin{abstract}
In receivers, IQ imbalances have significant effects on the performance of data transmission. Comparison of the performance of different types of Orthogonal Space-Time Block Codes $(O S T B C)$ in the presence of IQ imbalances show that different codes don't have a same behavior. Although most codes have an acceptable performance in low imbalances, they can't work properly in the presence of intensive non-idealities. Despite this general similarity, there are some differences in the behavior of the codes against non-idealities. Such differences make them suitable for different situations.
\end{abstract} tions

Keywords-MIMO; STBC; IQ imbalances; Mobile Communica-

\section{INTRODUCTION}

The ever-increasing demands for high-speed wireless data transmission has posed great challenges for wireless system designers to achieve high-throughput wireless communications in radio channels with limited bandwidth. Multiple transmit and receive antennas (MIMO) are most likely to be the dominant solution in future broadband wireless communication systems. This is because the capacity of a MIMO channel increases linearly with the minimum between the numbers of transmit and receive antennas in a richscattering environment without increasing the bandwidth and transmit power [1]-[4].

In wireless communications, frequency selective fading is the major undesired effect of the single-input single-output (SISO) which severely degrades the reliability and QOS of data transmission. Although this degradation was not considerable in the low speed and small communication networks, but it is one of the major challenges of the current and next generation high-speed networks. In order to overcome this problem, better use of the space by implementing space diversity and MIMO channels is suggested.

Low cost implementation of wireless systems is challenging in view of impairments associated with analog components. One such impairment is the mismatch between I and Q branches during down conversion of the received RF signal into baseband. These errors may severely degrade the quality of the received signal.

This paper is supported by Telecom. Co. of Chaharmahal va Bakhtiari
Space diversity is an applicable frequency efficient method. It is based on transmission of the same data stream through several independent spatial channels. Despite the ability of the space diversity to mitigate undesired transmission channel effects, it is not flexible enough to achieve arbitrary values of diversity and multiplexing gain. So it is not a good candidate for next generation communication systems. Using space-time codes [4] over MIMO channels is a proper suggestion for these systems due to their high flexibility in diversity and multiplexing gain. In fact, space diversity is a special case of space-time codes [1]. There are two type of space-time code namely Space-Time Trellis Code (STTC) and Space-Time Block Code (STBC) [4]. Here we focus on the second type of these codes.

In this paper, after introducing some of the mostly used sorts of the STBC codes, undesired effects of the RF-IF chain non-idealities on the performance of these codes are investigated.

The paper is organized as follows: Signal model, receiver architecture and IQ imbalances are introduced in section II. Various STBC codes and influence of the IQ imbalances on the Maximum Likelihood (ML) based decoders are discussed in section III and simulation results and performance compareson of different STBC codes are presented in section IV.

\section{RECEIVER STRUCTURE and SIGNAL MODEL}

Down-conversion is a fundamental stage in radio frequency front-end architecture in which the high carrier frequency signal is multiplied by local oscillating (LO) signals to be transferred to intermediate frequencies appropriate for further amplification and processing and eventually, to the zero frequency (baseband). There are different architectures to convert the RF signal to baseband, either through an intermediate frequency or by direct down-conversion to a baseband signal [5]. There are mainly two types of downconversions [6]: heterodyne receiver and zero intermediate frequency (ZIF). Due to certain advantages of direct conversion (cost, area, power consumption and less off-chip components), most of the future RF designs tend to adopt this scheme. Based on the architecture of the ZIF receiver (Fig. 1), regardless of the type of the elements, one can take the following imbalances into account as the total imbalances 
during the down-conversion process [9, 10]: 1. Amplitude error: difference between the amplitude of LO signals in I and $\mathrm{Q}$ branches. 2. Phase error: non-orthogonality of LO signals in I and Q branches. 3. DC offset: leakage of LO signal into RF port because of non perfect isolation between RF and LO inputs in I and Q branches. 4. Undesired signals: undesired RF signals transferred into the output because of non-ideal multiplication or imperfect filtering in I and Q branches.

Moreover, in each IQ branch different and independent imbalances will be accounted for.

To investigate the undesired effects of the above imbalances on the receiver output, a transmitted data sequence denoted as sn is assumed. The transmitted signal, $x(t)$, can be described as follows

$$
x(t)=A s_{n} g(t-n T) \cos \left(2 \pi f_{c} t\right)
$$

where $\mathrm{A}$ is the amplitude, $g(t)$ is the pulse shape and $\mathrm{fc}$ is the carrier frequency used for up-conversion. As a result of using multiple antennas at the receiver, several replicas of $\mathrm{x}(\mathrm{t})$ impaired by independent channel effects are received. In frequency domain, after an ideal down-conversion process, $r$ the $\mathrm{N} \times 1$ vector of the output can be shown as

$$
\mathbf{r}=\mathbf{h} s+\mathbf{n}
$$

where $\mathbf{h}=\left[\begin{array}{llll}h_{1} & h_{2} & \ldots & h_{N}\end{array}\right]^{T}$ and $\mathbf{n}=\left[\begin{array}{llll}\eta_{1} & \eta_{2} & \ldots & \eta_{N}\end{array}\right]^{T}$ are $\mathrm{N} \times 1$ channel and noise vectors, respectively and $\mathrm{s}$ is the frequency domain representation of the transmitted signal.

In order to enforce the down-conversion imbalances into the signal model, $\mathbf{r}^{\prime}$ a $\mathrm{N} \times 1$ vector is introduced as the output vector of the down-converters in discrete domain (after sampling)

$$
\mathbf{r}^{\prime}=\mathbf{h}^{\prime} \mathbf{s}+\mathbf{n}^{\prime}
$$

where $\mathbf{h}^{\prime}$ and $\mathbf{n}^{\prime}$ are the channel and noise vectors, respectively, affected by the down-conversion imbalances. That is $\mathbf{h}^{\prime}=\boldsymbol{\alpha} \otimes \mathbf{h}$ and $\mathbf{n}^{\prime}=\boldsymbol{\alpha} \otimes \mathbf{n}+\boldsymbol{\beta}$, where $\boldsymbol{\alpha}$ and $\boldsymbol{\beta}$ are $\mathrm{N} \times 1$ vectors. $\boldsymbol{\alpha}$ includes amplitude and phase errors and $\boldsymbol{\beta}$ represents DC offset and undesired signals. Also $\otimes$ is an element by element multiplication operator. Independent interelement IQ imbalances are also accounted for by using different elements for $\boldsymbol{\alpha}$ and $\boldsymbol{\beta}$.

As seen in the next section, ML based detection mostly used in decoding the STBC codes is based on the knowledge of the channel state information. So, changing this information may decrease the decoding performance.

\section{CODE STRUCTURE \& ML DETECTION TECHNIQUE}

In a general classification, space-time block codes are classified into orthogonal, quasi-orthogonal and nonorthogonal codes. The first sort benefits from simple ML decoding. Moreover the signal of each receive antenna is decoded separately, so one can use different number of receive antennas for an especial code. Because of these advantages, OSTBC is widely used in wireless communications devices in

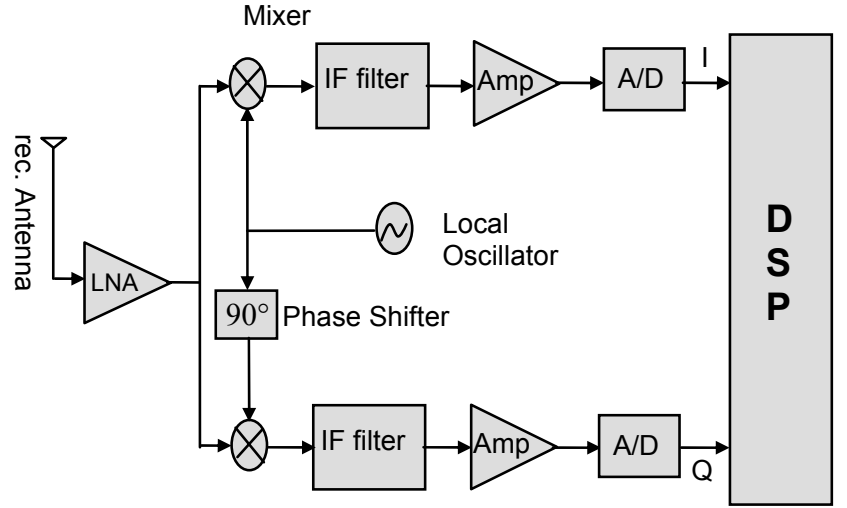

Mixer

Fig. 1. A simple view of ZIF receiver

which dimension, battery storage and weight are among the most important considerations [1].

Despite the advantages of OSTBC, it suffers from some limitations. In order to cover a broad range of wireless data

transmission, other sorts of the STBC's are established. QOSTBC is based on pair wise ML decoding. Compared to OSTBC, it supports more coding schemes. This code also let the receiver use arbitrary number of antennas.

In non-orthogonal STBC, relaxing the orthogonality of the code makes it applicable for a wide range of applications. The ability of non-orthogonal STBC to achieve different values of diversity, multiplexing gain and code rate is at the

expense of decoding complexity and dependence of the number of receive antennas on the coding scheme.

According to the characteristics of different STBC coding schemes and regarded to the constraints of portable devices in wireless communications, it seems that OSTBC has more capabilities to be implemented in wireless data transmission. There are several types of OSTBC schemes: Real OSTBC; Complex OSTBC; Generalized real OSTBC; Generalized complex OSTBC.

The first two types provide full diversity and are rate one schemes but they can only be used in a few situations. In order to overcome this limitation, the last two types are established. Although their rates are not as good as the first two types, they can cover more situations.

Each STBC code is introduced by its corresponding generation matrix. This matrix maps the input symbols into space and time i.e. it determines the transmitting symbols from different antennas in each time slot. Most properties of the code such as diversity, code gain, rate, complexity of the detector, number of transmit antennas and time delay are given by this matrix. As an example, (4) demonstrates the generation matrix of one of the mostly used complex orthogonal STBC's; Alamouti code [7].

$$
\mathbf{G}=\left(\begin{array}{cc}
x_{1} & x_{2} \\
-x_{2}^{*} & x_{1}^{*}
\end{array}\right)
$$




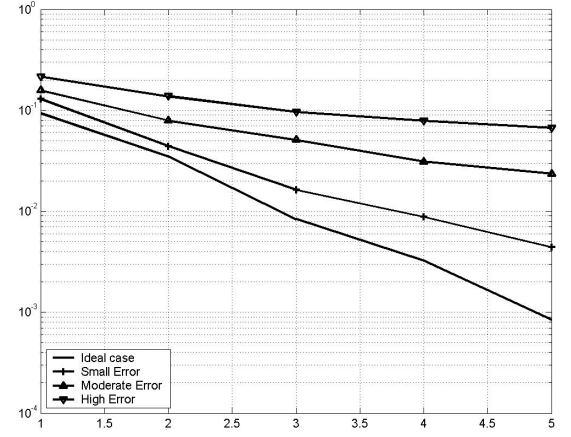

(a)

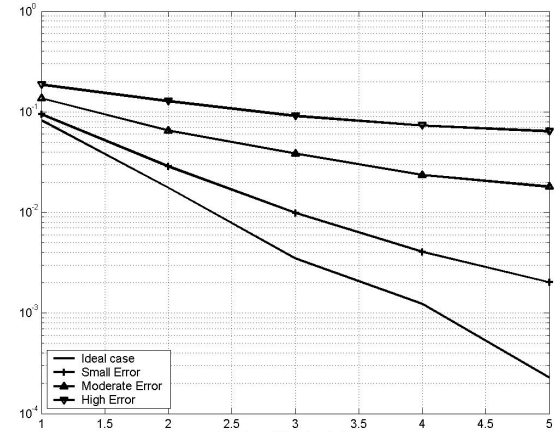

(b)

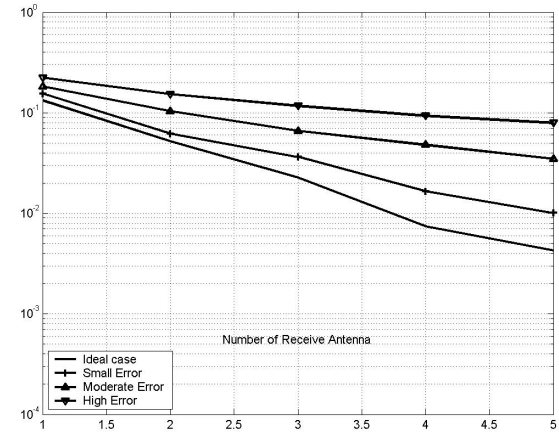

(c)

Fig.2. Impact of the IQ imbalances on the ML based decoding performance for three samples of real OSTBC's.

The curves demonstrate BER versus number of receive antenna

During the encoding process, code words are constructed by replacing the elements of $\mathrm{G}$ with proper number of transmitting symbols. Therefore, the transmitting code word is in the form of

$$
\mathbf{C}=\left(\begin{array}{cc}
s_{1}^{\prime} & s_{2}^{\prime} \\
-s_{2}^{\prime *} & s_{1}^{\prime *}
\end{array}\right)
$$

In general, each column and raw of this matrix determines the transmitting symbols by a special antenna and in a particular time slot. In other words, i,t-th element of this matrix is the symbol transmitted by $i$-th antenna in $t$-th timeslot. This process is to be continued until all symbols are encoded.

Equation (4) represents a rate one code which transmits two symbols in two time slots using two transmit antennas. Orthogonal STBC schemes benefit from two considerable advantages; simple ML decoding and separate decoding. Because of the second advantage, the decoding process and so the generation matrix are independent of the number of receive antennas. In other words, OSTBC can even be used with one receive antenna [2]. Of course in the presence of more than one receive antenna, maximum ratio combining (MRC) is used to combine different receiving versions of the signal.

It is assumed that the transmitted code word $\mathrm{C}$ is an OSTBC code passing through the channel with matrix $\mathrm{H}$ and received by $M$ antennas. The received signal can be demonstrated as:

$$
\mathbf{r}=\mathbf{C} \cdot \mathbf{H}+\mathbf{N}
$$

where $\mathrm{N}$ is the AWGN noise vector. Based on $\mathrm{ML}$ decoder, in each time slot the most similar symbol to the received signal is considered as the decoded symbol. It can be shown that in an ideal case, the output of the ML based detector $\hat{\mathbf{C}}$ is established as follows [1]:

$$
\hat{\mathbf{C}}=\underset{\mathbf{C}}{\arg \min }\left\{\sum_{m=1}^{M}\left[\mathbf{H}_{m}^{H} \cdot \mathbf{C}^{H} \cdot \mathbf{C} \cdot \mathbf{H}_{m}-2 \mathrm{R}\left(\mathbf{H}_{m}^{H} \cdot \mathbf{C}^{H} \cdot \mathbf{r}_{m}\right)\right]\right\}
$$

where $\mathrm{Hm}$ and $\mathrm{rm}$ are the $\mathrm{m}$-th columns of the channel matrix and the received signal matrix, respectively. In other words, $\mathrm{n}$-th element of $\mathrm{Hm}$ is the channel coefficient corresponding to $\mathrm{n}$-th transmit and $\mathrm{m}$-th receive antenna and $\mathrm{rm}$ is the received signal by the $\mathrm{m}$-th antenna. Therefore, the IQ imbalances which affect the received signal are unique for each rm and Hm. By taking the IQ imbalances into account, the received signal vector can be written as:

$$
\mathbf{r}_{m}=\mathbf{C} .\left(\mathbf{H}_{m} \otimes \mathbf{A}_{m}\right)+\mathbf{B}_{m}+\mathbf{N}_{m}
$$

where $\mathbf{A}_{m}=\left[a_{m t}\right]$ contain amplitude error, $\alpha_{m t}$, and phase error, $\varphi_{m t}$ as follows:

$$
a_{m t}=\left(1+\alpha_{m t}\right) e^{j \varphi_{m t}}
$$

$\mathbf{B}_{m}$ contains the last two IQ errors; LO leakage and undesired signals. Since $\mathbf{B}_{m}$ is uncorrelated with the transmitting signal. One can approximately model it as an ordinary noise which degrades the signal quality by decreasing the SNR (also confirmed by [8]). The first two errors in $\mathbf{A}_{m}$ not only affect the phase of the existing term, but also add an undesired term which is correlated with the desired one. Because of the complexity of the theoretical analysis, we continue our investigations in the next section by using computer simulations.

\section{SIMULATION RESULTS}

In this section, following OSTBC codes are investigated:

- $4 \times 4$ real orthogonal STBC with $r=1 \mathrm{sym} / \mathrm{sec}$ (Fig.2.a)

- $4 \times 3$ real generalized orthogonal STBC with $\mathrm{r}=0.75 \mathrm{sym} / \mathrm{sec}$ (Fig.2.b)

- $8 \times 5$ real generalized orthogonal STBC with $\mathrm{r}=1 \mathrm{sym} / \mathrm{sec}$ (Fig.2.c)

- $8 \times 4$ complex generalized orthogonal STBC with $\mathrm{r}=0.5 \mathrm{sym} / \mathrm{sec}$ (Fig.3.a)

- $2 \times 2$ complex Orthogonal STBC with $r=1 \mathrm{sym} / \mathrm{sec}$ (Alamouti code) (Fig.3.b) 


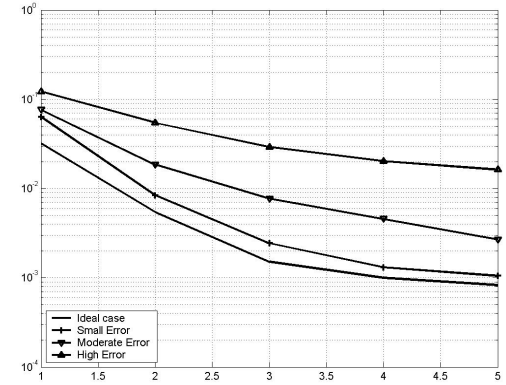

(a)

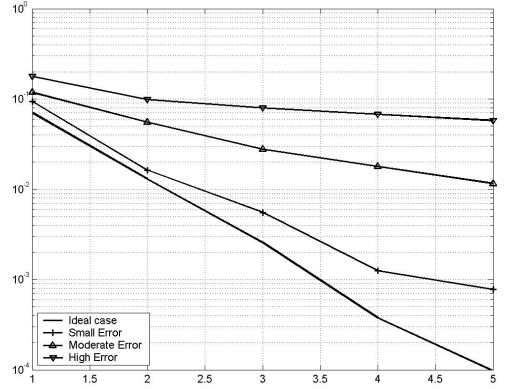

(b)

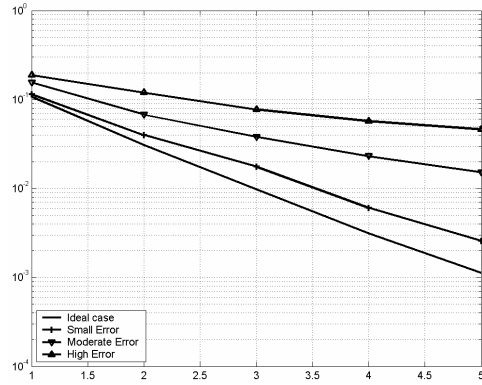

(c)

Fig.3. Impact of the IQ imbalances on the ML based decoding performance for three samples of complex OSTBC's.

The curves demonstrate BER versus number of receive antenna

- $4 \times 4$ complex generalized orthogonal STBC with $\mathrm{r}=$ $0.75 \mathrm{sym} / \mathrm{sec}$ (Fig.3.c).

While the number of receive antennas is the same for all simulations (five antennas), the number of transmit antennas is different. In order to have a fair justment, the total transmit power is considered to be the same for all codes. Therefore the more the number of transmit antennas, the less the power designated to each individual antenna. All simulations of this section represent Bit Error Rate (BER) of the received signal versus the number of receive antennas.

The codes used in the simulations of this section cover a wide range of OSTBC codes. Fig.2 and Fig3 show the performance of these codes. As seen in these figures, in case of ideal and low IQ imbalances, $2 \times 2$ Alamouti code has the best performance while the code used in Fig.3.a is the worst. On the other hand, in case of high IQ imbalances, the complex $8 \times 4$ generalized orthogonal STBC (Fig.3.a) has the best performance while the other codes are approximately the same.

Comparison of the performance of the codes shows that the behaviors of real codes are approximately the same as Alamouti code. They have an acceptable performance in low imbalances while significantly distorted in case of severe imbalances. The considerable difference between the first and last two curves shows that in case of rather high non-idealities, the BER of the received signal is degraded significantly whereas in other situations the imperfections are overcome by the OSTBC codes.

Besides the coding scheme, MRC combiner also influences the BER. The behavior of the curves with increasing the number of receive antennas shows the impact of the MRC method. As seen in the figures, the variations of the slope of the curves are approximately the same for different codes except the code of Fig.3.a. In this figure, in case of low imbalances and for more than three receive antennas the slope approaches to zero. It means that although this code has a better performance against severe imbalances, increasing the number of receive antennas can not improve the received signal quality, especially at low imbalances. Apart from this code, the improvement caused by increasing the number of receive antennas in other codes of Fig.2.b and Fig.3.b is considerable.

\section{CONCLUSION}

The relation of different OSTBC schemes in the presence of IQ imbalances is not the same. Despite of the general similarities, there are some differences between the behavior of the codes in terms of IQ imbalances intensities and the number of receive antennas.

It is shown that in low IQ imbalances, using OSTBC can improve the received signal quality. But the performance significantly degrades in the presence of severe non-idealities. Therefore, most OSTBC codes are efficient only at moderate imbalances. The importance of this problem rises in highspeed next generation mobile communications where challenges such as cost, dimension and weight are encountered. However one can still find suitable codes for different situations.

\section{REFERENCES}

[1] H. Jafarkhani, Space-Time Coding: Theory and practice Cambridge University Press, 2005.

[2] A.B. Gershman, N. D. Sidiropoulos, Space-Time Processing for MIMO Communications, John Wiley \& Sons Press, 2005.

[3] C. N. Chuah, D. N. C. Tse, J. M. Kahn, and R. A. Valenzuela, Capacity scaling in MIMO wireless systems under correlated fading, IEEE Trans. Inform. Theory, 48, 637-650, 2002.

[4] V. Tarohk, H. Jafarkhani, A. R. Calderbank, "Space-Time Block Codes From Orthogonal Designs," IEEE Trans. Information Theory, vol. 45, no. 5, July 1999, 1456-1467.

[5] A. Tarighat and A. H. Sayed, MIMO OFDM receivers for systems with IQ imbalances, IEEE Trans. Signal Processing, 53(9), 2006.

[6] R. A. Green, R. Aanerson-Sprecher, and J. W. Pierre, Quadrature Receiver Mismatch Calibration, IEEE Trans. Signal Processing, 47(11), $2000,3130-3133$.

[7] S. Alamouti, "A Simple transmitter Diversity scheme for Wireless Communications," IEEE Journal on Selected Areas in Communications, vol. 16, no. 8, Oct. 1998,1451-1458.

[8] B. Banitalebi, et.al., "Effects of the Transmission Channel on the LSCMA Based Beamformer in Mobile Communica-tions," Antem 2005, Saint Malo, France, June 15-17, 2005. 